\title{
Effect of Magnetohydrodynamics on Heat Transfer Behaviour of a Non-Newtonian Fluid Flow over a Stretching Sheet under Local Thermal Non-Equilibrium Condition
}

\author{
Konduru Sarada ${ }^{1}$, Ramanahalli J. Punith Gowda ${ }^{2}$ (D), Ioannis E. Sarris ${ }^{3, *} \mathbb{\text { , }}$, Rangaswamy Naveen Kumar ${ }^{2}(\mathbb{D})$ \\ and Ballajja C. Prasannakumara ${ }^{2}$ (I) \\ 1 Department of Mathematics, Government City College, Nayapul, Hyderabad 500002, India; \\ siddisarada@gmail.com \\ 2 Department of Studies and Research in Mathematics, Davangere University, \\ Davangere 577007, Karnataka, India; rjpunithgowda@gmail.com (R.J.P.G.); nkrmaths@gmail.com (R.N.K.); \\ dr.bcprasanna@gmail.com (B.C.P.) \\ 3 Department of Mechanical Engineering, University of West Attica, 12244 Athens, Greece \\ * Correspondence: ioannisarris@gmail.com
}

Citation: Sarada, K.; Gowda, R.J.P.; Sarris, I.E.; Kumar, R.N.; Prasannakumara, B.C. Effect of Magnetohydrodynamics on Heat Transfer Behaviour of a

Non-Newtonian Fluid Flow over a Stretching Sheet under Local Thermal Non-Equilibrium Condition. Fluids 2021, 6, 264. https://doi.org/10.3390/ fluids 6080264

Academic Editor: Francisco J

Galindo-Rosales

Received: 17 June 2021

Accepted: 22 July 2021

Published: 25 July 2021

Publisher's Note: MDPI stays neutral with regard to jurisdictional claims in published maps and institutional affiliations.

Copyright: (c) 2021 by the authors. Licensee MDPI, Basel, Switzerland. This article is an open access article distributed under the terms and conditions of the Creative Commons Attribution (CC BY) license (https:// creativecommons.org/licenses/by/ $4.0 /)$.

\begin{abstract}
A mathematical model is proposed to describe the flow, heat, and mass transfer behaviour of a non-Newtonian (Jeffrey and Oldroyd-B) fluid over a stretching sheet. Moreover, a similarity solution is given for steady two-dimensional flow subjected to Buongiorno's theory to investigate the nature of magnetohydrodynamics (MHD) in a porous medium, utilizing the local thermal nonequilibrium conditions (LTNE). The LTNE model is based on the energy equations and defines distinctive temperature profiles for both solid and fluid phases. Hence, distinctive temperature profiles for both the fluid and solid phases are employed in this study. Numerical solution for the nonlinear ordinary differential equations is obtained by employing fourth fifth order Runge-KuttaFehlberg numerical methodology with shooting technique. Results reveal that, the velocity of the Oldroyd-B fluid declines faster and high heat transfer is seen for lower values of magnetic parameter when compared to Jeffry fluid. However, for higher values of magnetic parameter velocity of the Jeffery fluid declines faster and shows high heat transfer when compared to Oldroyd-B fluid. The Jeffery liquid shows a higher fluid phase heat transfer than Oldroyd-B liquid for increasing values of Brownian motion and thermophoresis parameters. The increasing values of thermophoresis parameter decline the liquid and solid phase heat transfer rate of both liquids.
\end{abstract}

Keywords: local thermal non-equilibrium; magnetohydrodynamics (MHD); porous medium; OldroydB fluid; Jeffrey fluid; thermophoresis and Brownian motion

\section{Introduction}

Two basic models can be used for describing convective heat transport in a porous media: the local thermal equilibrium (LTE) model (one equation model) and the LTNE model (two equations model). The temperature difference among the liquid and solid phases are included in the LTNE model. In some circumstances, the assumption of LTE is not relevant if there is a considerable temperature difference between the two phases. At this stage, the LTNE model must be used, and an extra thermal boundary condition should be specified at the interface. Amiri et al. [1] initially proposed the two major approaches known as models $\mathrm{A}$ and $\mathrm{B}$ for the constant wall heat flux boundary constraints under the LTNE model in a completely filled porous medium channel. In the first method, the entire heat flux is divided between the liquid phase depending on their efficient conductivities and temperature gradients, whereas the second model approaches that each of the individual phases gets an equal share of the total heat flux. Later, using LTNE condition, numerous researchers explored the different liquid streams on diverse 
surfaces. Malashetty et al. [2] used the two-field model and the Lapwood-Brinkman model to construct an LTNE model to investigate convection in fluid with porous media. Muthtamilselvan et al. [3] explored the effect of LTNE on a laminar stream of nanofluid through an upright plate with permeable material. Prakash et al. [4] used the LTNE model to simulate the non-Darcian flow of liquid on an upright plate surrounded by a permeable material. Hashemi et al. [5] demonstrated the radiative flow of a micropolar nanoliquid within a porous medium under LTNE conditions.

The flow of a liquid on a stretching sheet (SS) in the occurrence of magnetic fields has received substantial attention because of its diverse applications in engineering processes such as glass fibre, plasma investigations, and geothermal energy extraction. During the past decades, numerous studies related to MHD effect on fluid flow over a SS have been published. Hayat et al. [6] conferred the MHD stagnancy flow of micropolar fluid past a non-linear SS. Mabood et al. [7] elucidated the entropy production analysis in MHD flow of Casson liquid past an SS. Aslani et al. [8] studied the radiative MHD stream of micropolar liquid on an SS. Kumar et al. [9] explored the magnetised flow of Casson nanoliquid on a coiled SS with chemical reaction. Radhika et al. [10] conferred the flow of dusty hybrid nanoliquid past an SS with melting and magnetic effect.

Nanofluids are liquids suspended with ultrafine particles that are diluted in nature. These fluids offer superior thermophysical characteristics over pure fluid carriers. The nanoparticle material and its volume fraction in the suspension have the greatest influence on the improvement of thermal conductivity. Buongiorno [11] discovered that the absolute velocity of a nanoparticle may be calculated by adding the relative velocity and base liquid velocity. He presented a nanofluid model for convection that included Brownian diffusion and thermophoresis factors into the energy equation. Later, using this model, numerous researchers explored the flow behaviour of different nanoliquids past various surfaces. Chu et al. [12] explored the MHD flow of third grade liquid on an SS using the Buongiorno model in presence of gyrotactic microbes. Using the Buongiorno model, Ijaz et al. [13] explored the entropy analysis in convective flow of Sisko liquid with Joule heating. Gowda et al. $[14,15]$ explored the Stefan blowing impact on flow of different liquids on an SS using Brownian diffusion and thermophoresis factors.

The flow and thermal analyses are commonly used in a variety of fields and have been researched by many scientists in numerous engineering applications, such as drying processes, thermal insulation, solar collectors, electronic cooling, heat exchangers, and chemical engineering. Recently, Lund et al. [16] exemplified the flow of $\mathrm{Cu}-\mathrm{Al}_{2} \mathrm{O}_{3} /$ water rotating hybrid nanofluid perfusing a porous medium over the stretching/shrinking surface. Irfan et al. [17] conferred the MHD flow of bio-nanoliquid on an SS with porous medium and radiation effect. Gowda et al. [18] explored the chemically reactive stream of second grade nanoliquid on an SS with Marangoni convection in the occurrence of a porous medium. Kumar et al. [19] studied the flow of Casson hybrid nanoliquid on a poignant moving disk in the presence of a porous medium. Tadesse et al. [20] exemplified the radiating stream of nanoliquid past a porous SS with magnetic effect.

The research of non-Newtonian liquids has piqued the interest of many researchers due to the wide range of uses in manufacturing and technical processes. In comparison to Newtonian fluids, the mechanics of flow, mass, and heat transport in non-Newtonian liquids is more complicated. As a result, the behaviour of non-Newtonian liquid motion is characterized in terms of strongly coupled and nonlinear equations, excluding the existence of closed form solutions. Because of their relaxation features, Jeffrey and Oldroyd-B nanofluids have important applications in fluid mechanics. Hence, several researchers have done a comparative study on these fluid flow models past diverse surfaces. Sandeep et al. [21] did a comparative analysis on Maxwell, Oldroyd-B, and Jeffrey fluid flows on a porous SS using the Buongiorno model. In the presence of a thermal radiation, transverse magnetic field, non-uniform heat source and sink, Sandeep and Sulochana [22] introduced a novel model for investigating the heat transfer behaviour of Maxwell, Oldroyd-B, and Jeffrey nanofluids on an SS. The flow of nano-fluid models like Maxwell, Oldroyd-B, and Jeffery with heat 
source/sink across a cone was examined by Reddy et al. [23]. The entropy production was examined by Almakki et al. [24] with Brownian movement and thermophoresis diffusions by utilizing Maxwell, Oldroyd-B, and Jeffery nanofluid models. Saeed et al. [25] examined the chemically reacting MHD flow of Maxwell, Oldroyd-B, and Jeffery nanofluid models with modified Fourier heat flux.

The present investigation demonstrates that the intensity of the LTNE for the phenomena of heat transfer and flow of Jefferey and Oldroyd-B liquids in the presence of porous media and MHD is not effectively covered. Furthermore, several relevant characteristics that affect heat transfer improvement are not taken into account. As a result, the purpose of this research is to demonstrate the influence of dimensionless factors on the involved profiles of Jefferey and Oldroyd-B nanomaterial liquids using the Buongiorno model. In addition, we carried out a comparison analysis of Jefferey and Oldroyd-B nanomaterial liquids.

\section{Mathematical Formulation}

Consider a steady incompressible laminar flow of two combined non-Newtonian nanofluids (Oldroyd-B and Jeffrey) over a sheet being stretched with the velocity $U_{w}=c x$ in a porous medium. The flow being restricted to $y>0$ (see Figure 1 ) and coinciding with the plane $y=0$. Further, the mathematical model used for the nanofluid is the one which incorporates the effects of Brownian motion and thermophoresis. Homogeneousness in the porous medium is assumed. It is also assumed that the magnetic field $B_{0}$ is applied perpendicular to the sheet. The heat transfer analysis of the liquid and solid phase is analysed using two different heat transport equations [4,26]:

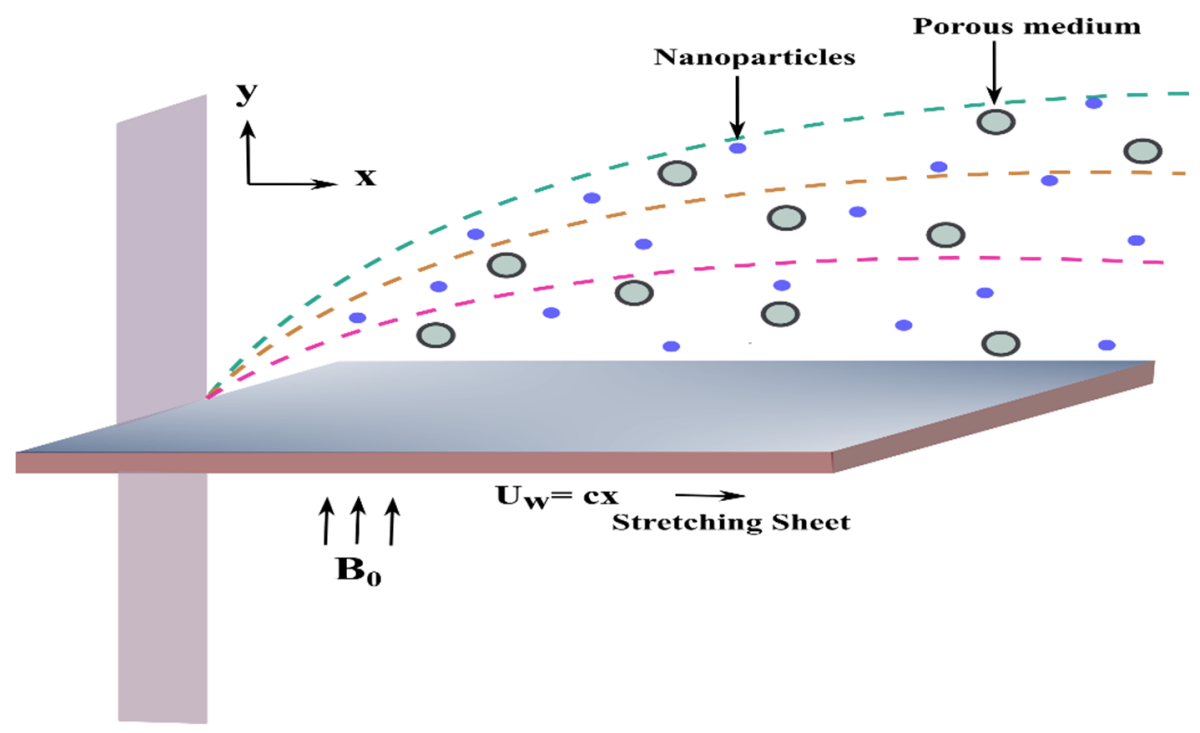

Figure 1. Flow geometry.

The equations for the assumed flow, heat, and mass transfer scrutiny inside the porous medium takes the following forms $[4,21,23]$ :

$$
\begin{gathered}
\frac{\partial u}{\partial x}+\frac{\partial v}{\partial y}=0 \\
\frac{1}{\varepsilon^{2}}\left[u \frac{\partial u}{\partial x}+v \frac{\partial u}{\partial y}\right]+\beta_{1}\left[u^{2} \frac{\partial^{2} u}{\partial x^{2}}+v^{2} \frac{\partial^{2} u}{\partial y^{2}}+2 u v \frac{\partial^{2} u}{\partial x \partial y}\right]= \\
\frac{\mu_{f}}{\rho_{f}\left(1+\beta_{2}\right)}\left(\frac{\partial^{2} u}{\partial y^{2}}+\beta_{3}\left(u \frac{\partial^{3} u}{\partial x \partial y^{2}}+v \frac{\partial^{3} u}{\partial y^{3}}-\frac{\partial u}{\partial x} \frac{\partial^{2} u}{\partial y^{2}}+\frac{\partial u}{\partial y} \frac{\partial^{2} u}{\partial x \partial y}\right)\right) \\
-\frac{\mu_{f}}{\rho_{f}} K_{K^{*}}-\frac{\sigma_{f} B_{0}^{2}}{\rho_{f}} u,
\end{gathered}
$$




$$
\begin{gathered}
\frac{1}{\varepsilon}\left[u \frac{\partial T_{f}}{\partial x}+v \frac{\partial T_{f}}{\partial y}\right]=\frac{k_{f}}{\left(\rho C_{p}\right)_{f}}\left(\frac{\partial^{2} T_{f}}{\partial y^{2}}+\frac{\partial^{2} T_{f}}{\partial x^{2}}\right) \\
\left.+\tau\left[D_{B} \frac{\partial C}{\partial y} \frac{\partial T_{f}}{\partial y}+\frac{D_{T}}{T_{\infty}}\left(\frac{\partial T_{f}}{\partial y}\right)^{2}\right]+\frac{h_{f s}}{\varepsilon\left(\rho C_{p}\right)_{f}}\left(T_{s}-T_{f}\right),\right\} \\
0=\frac{k_{s}}{\left(\rho C_{p}\right)_{s}}\left(\frac{\partial^{2} T_{s}}{\partial y^{2}}+\frac{\partial^{2} T_{s}}{\partial x^{2}}\right)+\frac{h_{f s}}{(1-\varepsilon)\left(\rho C_{p}\right)_{s}}\left(T_{f}-T_{s}\right), \\
\frac{1}{\varepsilon}\left[u \frac{\partial C}{\partial x}+v \frac{\partial C}{\partial y}\right]=D_{B}\left(\frac{\partial^{2} C}{\partial y^{2}}+\frac{\partial^{2} C}{\partial x^{2}}\right)+\frac{D_{T}}{T_{\infty}}\left(\frac{\partial^{2} T_{f}}{\partial y^{2}}\right) .
\end{gathered}
$$

The imposed boundary constraints are:

$$
\left.\begin{array}{c}
u=c x, v=0, T_{f}=T_{w}, T_{p}=T_{w}, C=C_{w} \text { at } y=0, \\
u \rightarrow 0, \frac{\partial u}{\partial y} \rightarrow 0, T_{f} \rightarrow T_{\infty}, T_{p} \rightarrow T_{\infty}, C \rightarrow C_{\infty} \text { as } y \rightarrow \infty .
\end{array}\right\}
$$

The proposed mathematical model is deliberated based on the following conditions:

i. The problem represents Jeffrey fluid, if $\beta_{1}=0, \beta_{2} \neq 0, \beta_{3} \neq 0$.

ii. The problem represents Oldroyd-B fluid, if $\beta_{1} \neq 0, \beta_{2}=0, \beta_{3} \neq 0$.

Dimensionless quantities employed in the mathematical model are as follows:

$$
\left.\begin{array}{l}
\psi=\sqrt{\mathcal{C V} v_{f}} x f(\eta), u=\frac{\partial \psi}{\partial y}=c x f^{\prime}(\eta), v=-\frac{\partial \psi}{\partial x}=-\sqrt{\mathcal{C V} v_{f}} f(\eta), \eta=\sqrt{\frac{c}{v_{f}}} y, \\
\theta_{f}(\eta)=\frac{T_{f}-T_{\infty}}{T_{w}-T_{\infty}}, \theta_{s}(\eta)=\frac{T_{s}-T_{\infty}}{T_{w}-T_{\infty}}, \chi(\eta)=\frac{C-C_{\infty}}{C_{w}-C_{\infty}} .
\end{array}\right\}
$$

Using Equation (7), the governing Equations (2)-(5) are reduced as follows:

$$
\left.\begin{array}{c}
f^{\prime \prime \prime}+\lambda_{2}\left[f^{\prime \prime 2}-f f^{i v}\right]-\frac{\left(1+\beta_{2}\right)}{\varepsilon^{2}}\left[f^{2}-f f^{\prime \prime}\right]-\left(1+\beta_{2}\right) K^{* *} f^{\prime} \\
-\left(1+\beta_{2}\right) M f^{\prime}-\left(1+\beta_{2}\right)\left[\lambda_{1}\left(f^{2} f^{\prime \prime \prime}-2 f f^{\prime} f^{\prime \prime}\right)\right]=0,
\end{array}\right\}
$$

along with the associated reduced boundary constraints:

$$
\left.\begin{array}{l}
f^{\prime}(0)=1, f(0)=0, \theta_{f}(0)=1, \theta_{s}(0)=1, \chi(0)=1 \\
f^{\prime}(\infty) \rightarrow 0, f^{\prime \prime}(\infty) \rightarrow 0, \theta_{f}(\infty) \rightarrow 0, \theta_{s}(\infty) \rightarrow 0, \chi(\infty) \rightarrow 0 .
\end{array}\right\}
$$

The emerging non-dimensional physical parameters in the model are as follows:

$$
\begin{aligned}
& K^{* *}=\frac{v_{f}}{K^{*} c}, \lambda_{1}=\beta_{1} c, \lambda_{2}=\beta_{3} c, \operatorname{Pr}=\frac{v_{f}}{\alpha_{f}}, S c=\frac{v_{f}}{D_{B}}, M=\frac{\sigma_{f} B_{0}^{2}}{\rho_{f} c}, \\
& N_{t}=\frac{\tau D_{T}\left(T_{w}-T_{\infty}\right)}{v_{f} T_{\infty}}, N_{b}=\frac{\tau D_{B}\left(C_{w}-C_{\infty}\right)}{v_{f}}, \gamma=\frac{\varepsilon k_{f}}{(1-\varepsilon) k_{s}}, H=\frac{h_{f s} \alpha_{f}}{\varepsilon c k_{f}} .
\end{aligned}
$$

The local skin friction, Nusselt number, and Sherwood number relations are used to determine the resistance to flow, rate of heat and mass transfer. These relations in their non-dimensional form takes the following structure:

$$
\begin{gathered}
\sqrt{\operatorname{Re}} C_{f}=2\left(\frac{1+\lambda_{1}}{1+\lambda_{2}}\right) f^{\prime \prime}(0), \\
\frac{N u_{f}}{\sqrt{\operatorname{Re}}}=-\theta^{\prime}{ }_{f}(0),
\end{gathered}
$$




$$
\begin{aligned}
& \frac{N u_{s}}{\sqrt{\operatorname{Re}}}=-\theta^{\prime}{ }_{s}(0), \\
& \frac{S h}{\sqrt{\operatorname{Re}}}=-\chi^{\prime}(0) .
\end{aligned}
$$

\section{Result and Discussion}

This section features a detailed explanation as well as the influence of important relevant factors on the related distributions. The impact of these characteristics is presented visually one by one and is explained clearly in terms of physical importance. All calculations are performed for an extensive array of parameters involved in the flow problem. The system of modelled equations is reduced by using suitable similarity transformations, which are then tackled numerically by using Runge-Kutta-Fehlberg-45 (RKF-45) process with shooting technique. The influence of dimensionless factors on the flow, heat, and mass transfer of Jefferey and Oldroyd-B nanomaterial liquids are analysed using the Buongiorno model. In addition, we carried out a comparison analysis of Jefferey and Oldroyd-B nanomaterial liquids. The Prandtl number has been set to be adjusted for all of this, while other parameters have been set to be varied in order to analyse their effects on velocity, mass and heat transport. We also compared the obtained numerical results to published work in Table 1 and found a good agreement with each other.

Table 1. Comparison of results for the thermal gradient with published papers for some reduced cases.

\begin{tabular}{cccccccc}
\hline & \multicolumn{4}{c}{ Published Papers $\boldsymbol{\theta}^{\prime}(0)$} & \multicolumn{2}{c}{ Present Results } \\
\cline { 2 - 5 } Pr & \multirow{2}{*}{ Ishak et al. [27] } & \multirow{2}{*}{ Vajravelu et al. [28] } & \multicolumn{2}{c}{ Prakash et al. [4] } & \multirow{2}{*}{$\boldsymbol{\theta}_{\boldsymbol{s}}^{\prime}(0)$} & $\boldsymbol{\theta}_{\boldsymbol{f}}^{\prime}(0)$ \\
& & & 0.808836 & 0.808570 & 0.808578 & 0.808619 & 0.808625 \\
\hline 0.7 & 0.8086 & 1.000000 & 0.999927 & 0.999937 & 0.999945 & 0.999953 \\
\hline 1 & 1.0000 & 1.923687 & 1.923556 & 1.923573 & 1.923675 & 1.923685 \\
\hline 2 & 1.9237 & 3.720788 & 3.720444 & 3.720475 & 3.720616 & 3.720632 \\
\hline 10 & 3.7207 & & & & &
\end{tabular}

Figure 2a reveals the domination of $K^{* *}$ on $f^{\prime}(\eta)$ for both Oldroyd-B and Jefferey liquids. The improvement in $K^{* *}$ weakens the $f^{\prime}(\eta)$ of both liquids. The increasing values of porosity component improve the system's resistance. Because of the higher frictional force, this produces a reduction in fluid flow. In this case, increased porosity increases the surface's resistance to liquid motion. This increased resistance reduces the velocity of the liquid. Further, for lower values of $K^{* *}, f^{\prime}(\eta)$ for Oldroyd-B liquid is strongly triggered and declines faster than Jefferey liquid. However, for higher values of $K^{* *}$, the $f^{\prime}(\eta)$ of Jeffery liquid is strongly triggered and declines faster than Oldroyd-B liquid. Figure $2 \mathrm{~b}$ is plotted to disclose the domination of $K^{* *}$ on $\theta_{f}(\eta)$ for both Oldroyd-B and Jefferey liquids. The escalation in $K^{* *}$ improves the $\theta_{f}(\eta)$ for both Oldroyd-B and Jefferey liquids. With a rise in $K^{* *}$, the fluids become more viscous, and the fluid's velocity stagnates as a consequence of the higher viscosity that restricts fluid mobility over the surface, the $K^{* *}$ indicates resistance to movement as the temperature gradient increases. This is consistent with the notion that raising $K^{* *}$ causes an increase in tension, which is responsible for temperature boundary layer thickening. It is obvious that the occurrence of a permeable material causes progressive constraint to the flow of liquid, resulting in a diminution in liquid velocity and a rise in heat transfer. Further, it is noticed that Oldroyd-B liquid shows improved heat transfer compared to Jefferey liquid for lower porosity, but for improved porosity values, Jefferey liquid shows improved heat transfer compared to Oldroyd-B liquid. 

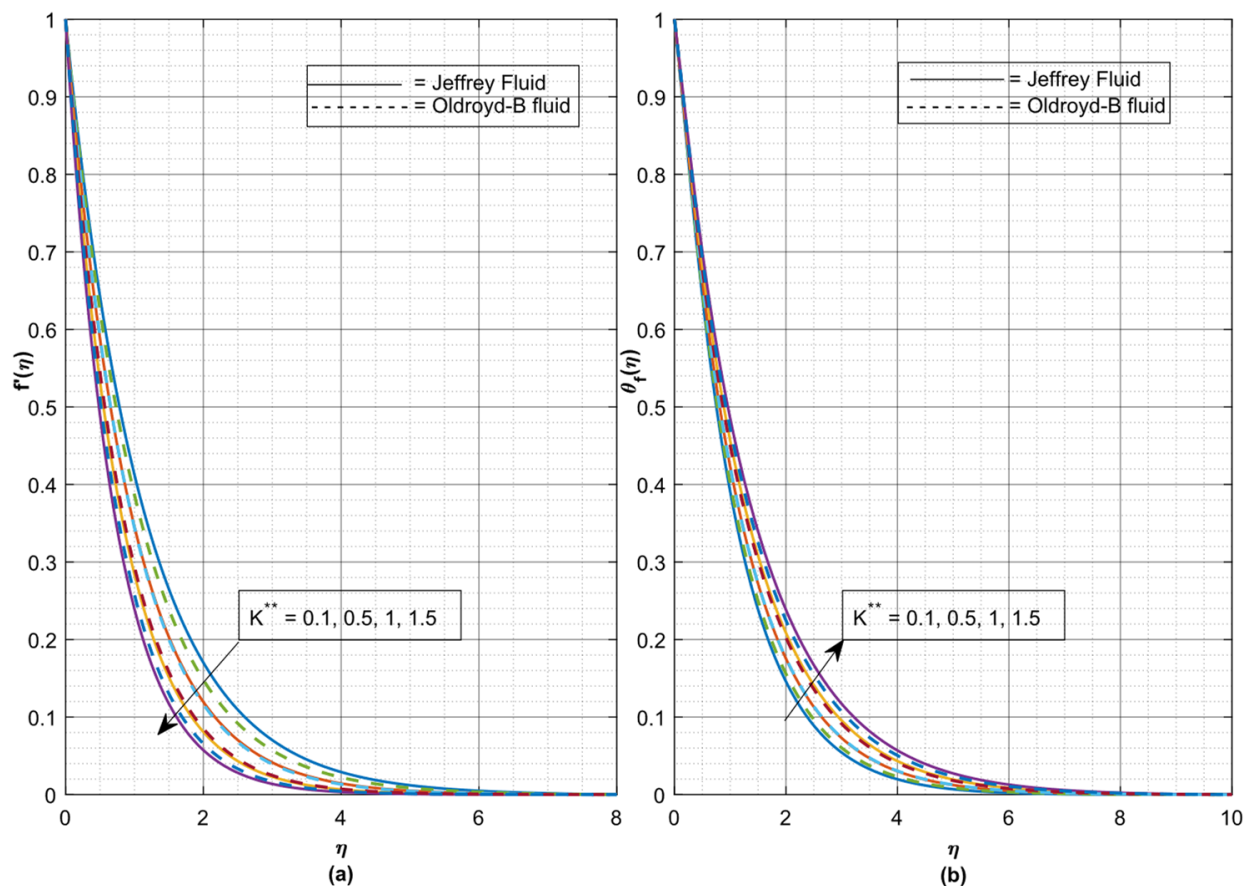

Figure 2. (a) Impact of $K^{* *}$ on $f^{\prime}(\eta)$ and (b) Impact of $K^{* *}$ on $\theta_{f}(\eta)$.

Figure 3a is plotted to explore the domination of $M$ on $f^{\prime}(\eta)$ for both Oldroyd-B and Jefferey liquids. The improvement in $M$ weakens the $f^{\prime}(\eta)$ of both liquids. The magnetic field's influence on the flow field is indicated by the $M$. The presence of a magnetization in the fluid flow region causes the fluid to move more slowly. In general, an increase in the $M$ produces the opposite force to the flow, which is known as the Lorentz force. This force tends to diminish the thickness of the velocity boundary layer. Further, for lower values of $M, f^{\prime}(\eta)$ for Oldroyd-B liquid is strongly triggered and declines faster than Jefferey liquid. However, for higher values of $M$, the $f^{\prime}(\eta)$ of Jeffery liquid is strongly triggered and declines faster than Oldroyd-B liquid. The stimulation of $M$ on $\theta_{f}(\eta)$ for both liquids is exemplified in Figure $3 \mathbf{b}$. The escalating values of $M$ decays the $\theta_{f}(\eta)$ of both liquids. According to these observations, the magnetic force adds another layer of resistance to the flow, slowing velocity and increasing temperature. The existence of a magnetic field induces Lorentz force, resulting in a retarding effect on nanoparticles and the base fluid velocity field. Thermal energy is released as a consequence of the extra effort required to draw the nanofluid toward the operation of the magnetic field. This heats the fluid, raising fluid temperatures. Further, it is noticed that Oldroyd-B liquid shows improved heat transfer compared to Jefferey liquid for lower $M$ values, but for improved $M$ values, Jefferey liquid shows improved heat transfer compared to Oldroyd-B liquid.

Figure 4 a shows the power of $\gamma$ on $\theta_{f}(\eta)$ of both liquids. The growth in $\gamma$ declines the $\theta_{f}(\eta)$ of both liquids. According to the definition of the $\gamma$, the ability of the fluid phase for heat transfer decreases as $\gamma$ increases. Moreover, fluid phase heat transfer of Jefferey liquid is more than that of fluid phase heat transfer of Oldroyd-B liquid and decays slowly for growing values of $\gamma$. Figure $4 \mathrm{~b}$ shows the impact of $\gamma$ on $\theta_{s}(\eta)$ of both Oldroyd-B and Jefferey liquids. The growth in $\gamma$ declines the $\theta_{s}(\eta)$ of both Oldroyd-B and Jefferey liquids. Increasing $\gamma$ causes the system to become unstable. The influence is most obvious when there is very little $\gamma$. Physically, at higher $\gamma$ values, convection may be completely suppressed, resulting in declination of both fluid and solid phase thermal profiles. Moreover, solid phase heat transfer of Jefferey liquid is more than that of solid phase heat transfer of Oldroyd-B liquid and decays slowly for growing values of $\gamma$. 


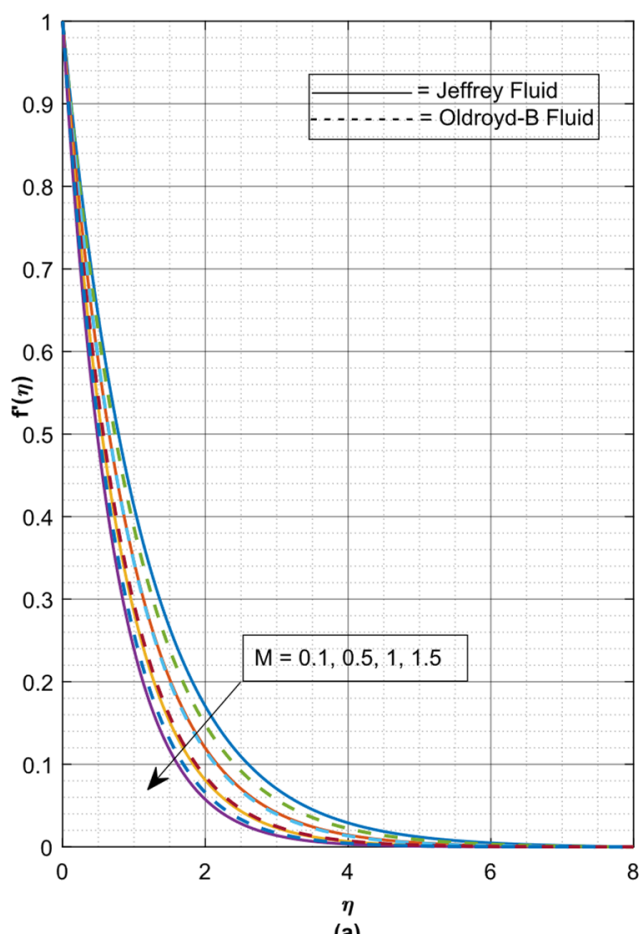

(a)

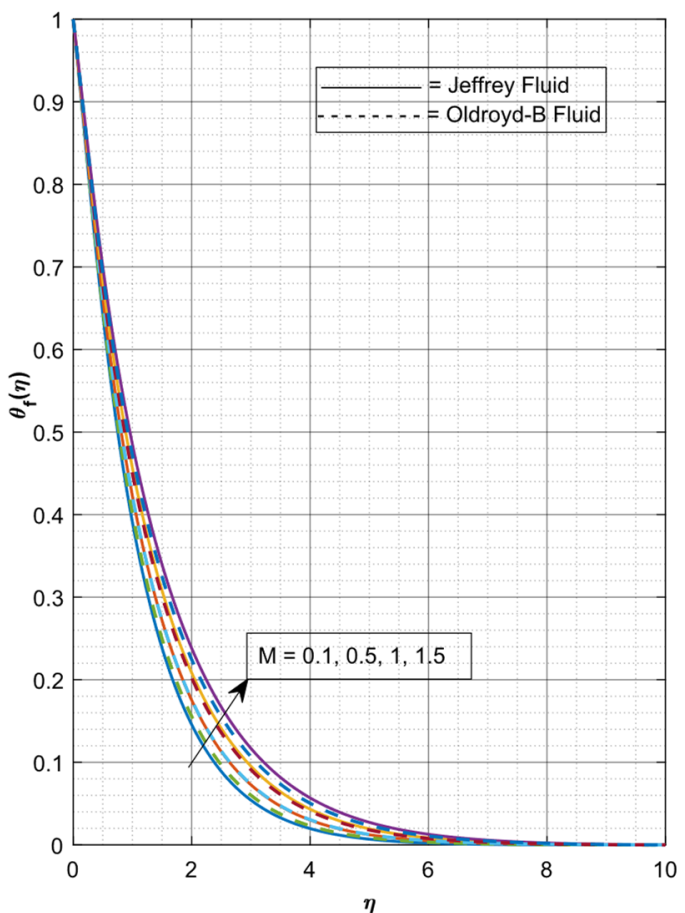

(b)

Figure 3. (a) Impact of $M$ on $f^{\prime}(\eta)$ and (b) Impact of $M$ on $\theta_{f}(\eta)$.

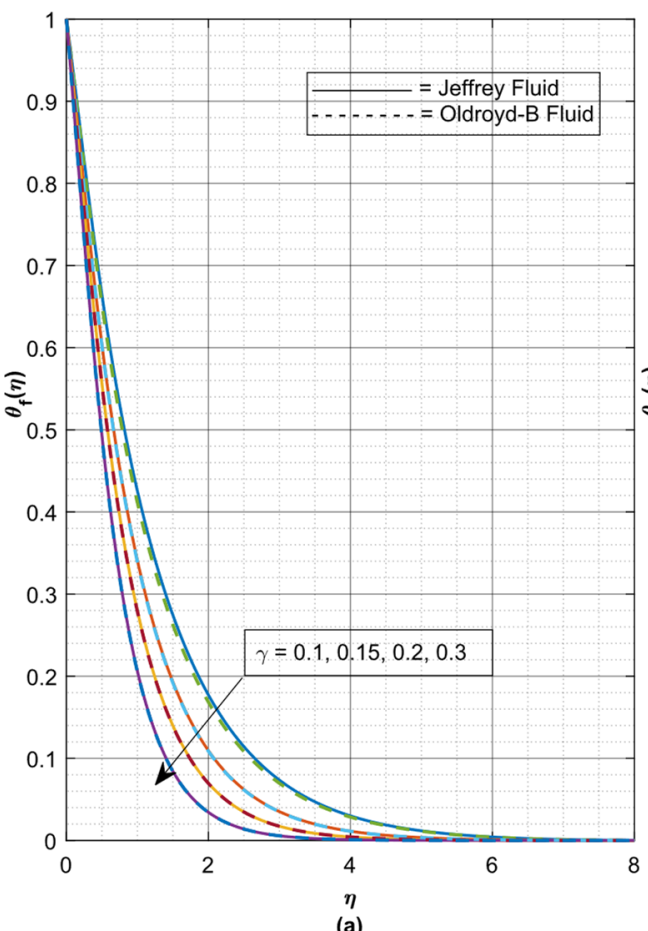

(a)

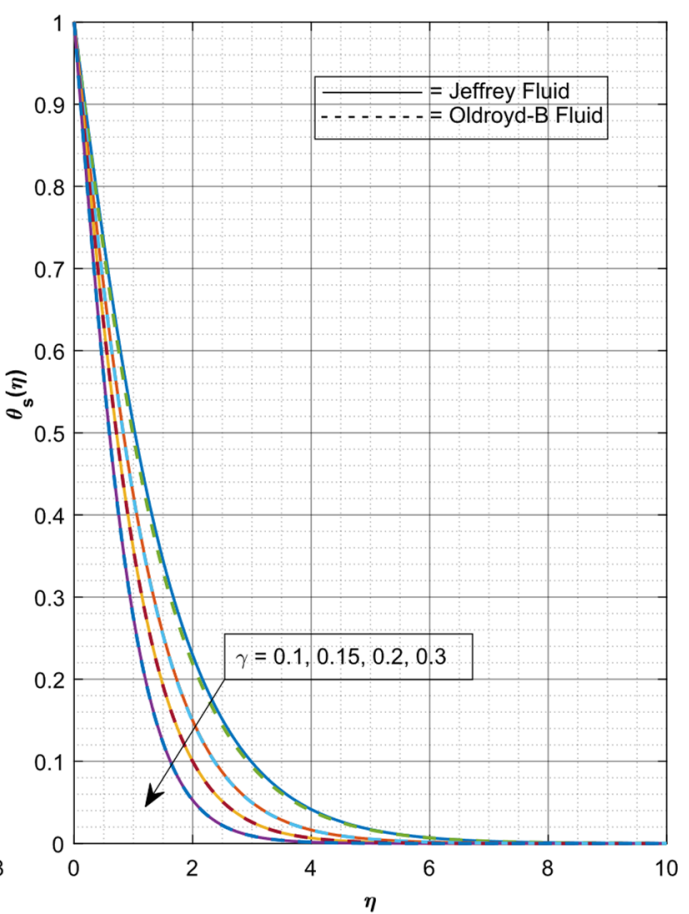

(b)

Figure 4. (a) Impact of $\gamma$ on $\theta_{f}(\eta)$ and (b) Impact of $\gamma$ on $\theta_{s}(\eta)$.

Figure 5a shows the stimulation of $H$ on $\theta_{f}(\eta)$ of both Oldroyd-B and Jefferey liquids. The growth in $H$ rises the $\theta_{f}(\eta)$ of both Oldroyd-B and Jefferey liquids. Further, both liquids show approximately the same fluid phase heat transfer for escalating values of $H$. Figure $5 \mathrm{~b}$ displays the stimulation of $H$ on $\theta_{s}(\eta)$ of both Oldroyd-B and Jefferey liquids. The growth in $H$ declines the $\theta_{s}(\eta)$ of both liquids. Further, both liquids show approximately the same solid phase heat transfer for escalating values of $H$. Here, the thermal gradient of 
the solid phase drops and liquid phase upsurges as $H$ increases due to fast heat transfer between the phases.
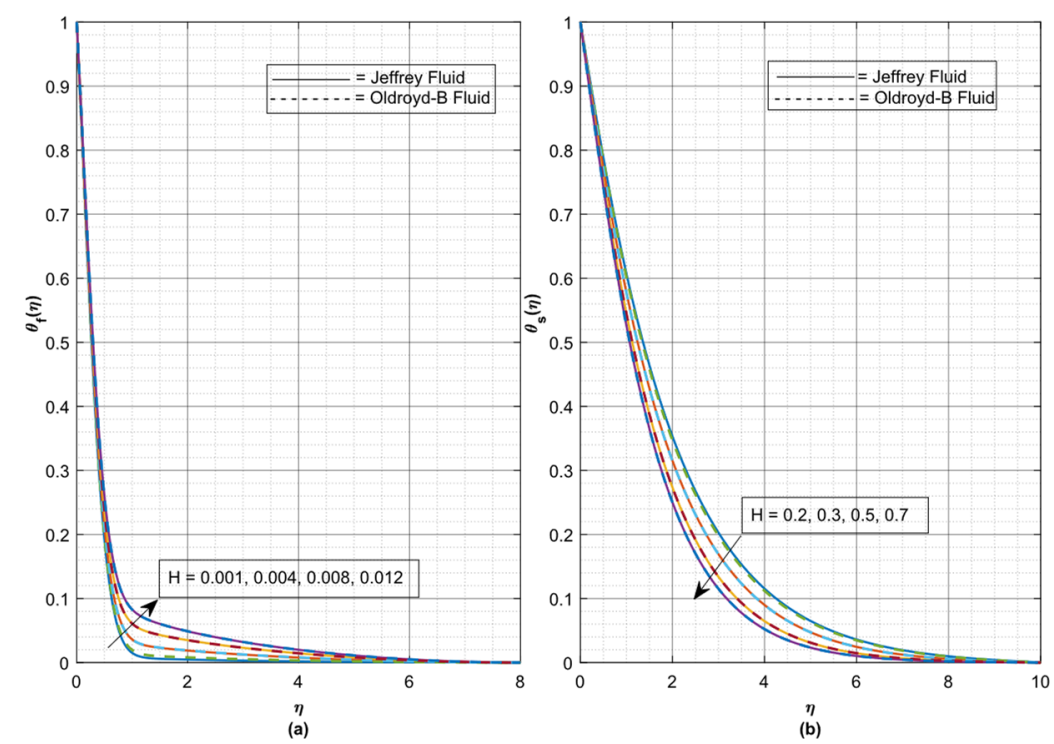

Figure 5. (a) Impact of $H$ on $\theta_{f}(\eta)$ and (b) Impact of $H$ on $\theta_{S}(\eta)$.

Figure 6a shows the stimulation of $N_{t}$ on $\theta_{f}(\eta)$ of both Oldroyd-B and Jefferey liquids. The growth in $N_{t}$ rises the $\theta_{f}(\eta)$ of both Oldroyd-B and Jefferey liquids. Physically, as the thermophoretic effect becomes stronger, high thermal conductivity nanoparticles penetrate deeper into the fluid, increasing the thickness of the thermal boundary layer. Under a temperature gradient, nanoparticles prefer to travel from warm to cold zones. The profiles get larger when the $N_{t}$ is increased, showing a rise in thermal boundary layer thickness. Further, Jeffery liquid show improved fluid phase heat transfer compared to Oldroyd-B liquid for escalating values of $N_{t}$. Figure $6 \mathrm{~b}$ shows the stimulation of $N_{b}$ on $\theta_{f}(\eta)$ of both Oldroyd-B and Jefferey liquids. The growth in $N_{b}$ rises the $\theta_{f}(\eta)$ of both Oldroyd$B$ and Jefferey liquids. Further, Jeffery liquid show improved fluid phase heat transfer compared to Oldroyd-B liquid for higher values of $N_{b}$. This is owing to the fact that various nanoparticles have varied $N_{b}$ values, which causes the $\theta_{f}(\eta)$ for liquid to increase.
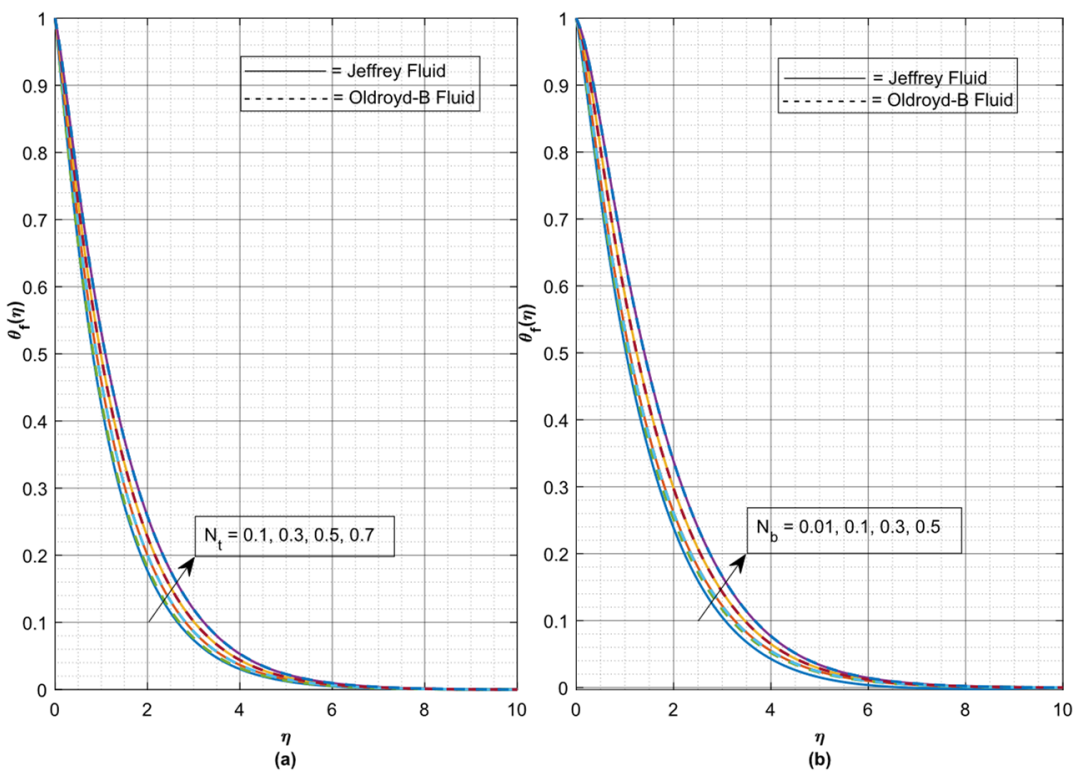

Figure 6. (a) Impact of $N_{t}$ on $\theta_{f}(\eta)$ and (b) Impact of $N_{b}$ on $\theta_{f}(\eta)$. 
Figure 7a shows the stimulation of $N_{t}$ on $\chi(\eta)$ of both Oldroyd-B and Jefferey liquids. The growth in $N_{t}$ rises the $\chi(\eta)$ of both Oldroyd-B and Jefferey liquids. $N_{t}$ denotes the ratio of nanoparticle diffusion via thermophoretic force to momentum diffusion in nanoliquid. The profiles get larger when the $N_{t}$ is increased, showing an increase in solutal boundary layer thickness. Further, Jeffery liquid shows improved fluid phase mass transfer compared to Oldroyd-B liquid for higher values of $N_{t}$. The impact of $S c$ on $\chi(\eta)$ of both Oldroyd-B and Jefferey liquids is showed in Figure $7 \mathrm{~b}$. The gain in $S c$ decay the mass transfer. The growing value of $S c$ increases momentum diffusivity and causes the mass transport to deteriorate. Schmidt number is a dimensionless number that describes the relationship between mass and momentum diffusivities in a fluid flow. The hydrodynamic thickness layer and the mass transfer layer are the physical names for these two concepts. The smallest $S c$ refers to the highest concentration of the nanoparticles. Further, the $\chi(\eta)$ for Jefferey liquid decays slower than that of the Oldroyd-B liquid for escalating values of $S c$.

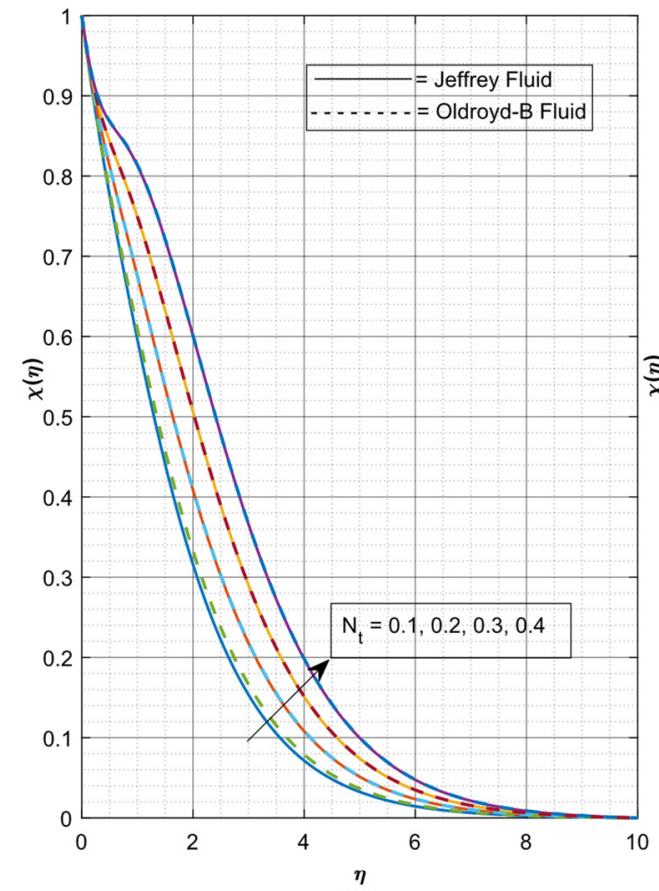

(a)

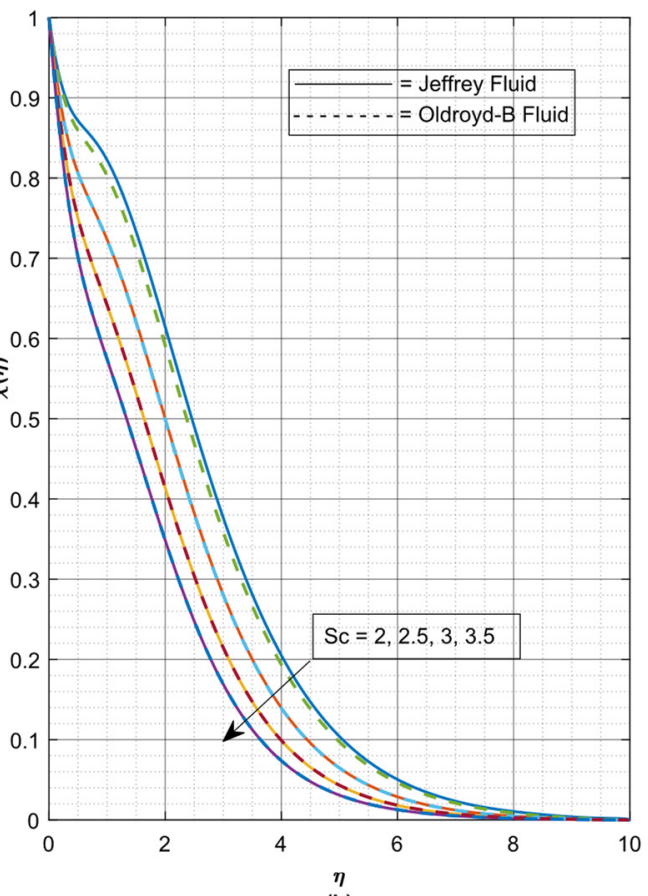

(b)

Figure 7. (a) Impact of $N_{t}$ on $\chi(\eta)$ and (b) Impact of $S c$ on $\chi(\eta)$.

The impact of $N_{t}$ on $\operatorname{Re}^{-1 / 2} N u_{f}$ versus $\gamma$ for both liquids is exemplified in Figure 8a. The escalating values of $N_{t}$ deteriorations the $\operatorname{Re}^{-1 / 2} N u_{f}$ of both liquids, but a contrary trend is seen for improved $\gamma$ values. Moreover, Oldroyd-B liquid shows a slightly improved fluid phase heat transfer rate compared to Jeffrey liquid for growing values of $N_{t}$. The encouragement of $N_{t}$ on $\operatorname{Re}^{-1 / 2} N u_{s}$ versus $\gamma$ for both nanoliquids is exemplified in Figure $8 b$. The escalating values of $N_{t}$ declines the $\operatorname{Re}^{-1 / 2} N u_{s}$ of both liquids. However, inverse movement is seen for improved values of $\gamma$. Moreover, Jeffrey liquid shows improved solid phase heat transfer rate compared to Oldroyd-B liquid for growing values of both $N_{t}$ and $\gamma$. From Figure 8a,b we conclude that, an upsurge in the $H$ leads to growth in the solid phase heat transfer rate of Jeffrey liquid while it declines the fluid phase heat transference rate of Jeffrey fluid. Figure 9 a depicts the impact of $M$ on $\operatorname{Re}^{1 / 2} C_{f}$ versus $K^{* *}$ for both liquids. The escalating values of $M$ and $K^{* *}$ decays the $\operatorname{Re}^{1 / 2} C_{f}$ of both liquids. Moreover, $\operatorname{Re}^{1 / 2} C_{f}$ for Jeffrey liquid declines faster than that of the Oldroyd-B liquid. The change in $S h R^{-1 / 2}$ versus $S c$ for escalating values of $N_{b}$ for both liquids is revealed in Figure $9 \mathrm{~b}$. Here, $S h R e^{-1 / 2}$ improves for booming values of both $N_{b}$ and $S c$. Moreover, 
Jeffrey liquid shows more mass transfer rate than that of the Oldroyd-B liquid for rising values of both $N_{b}$ and $S c$.

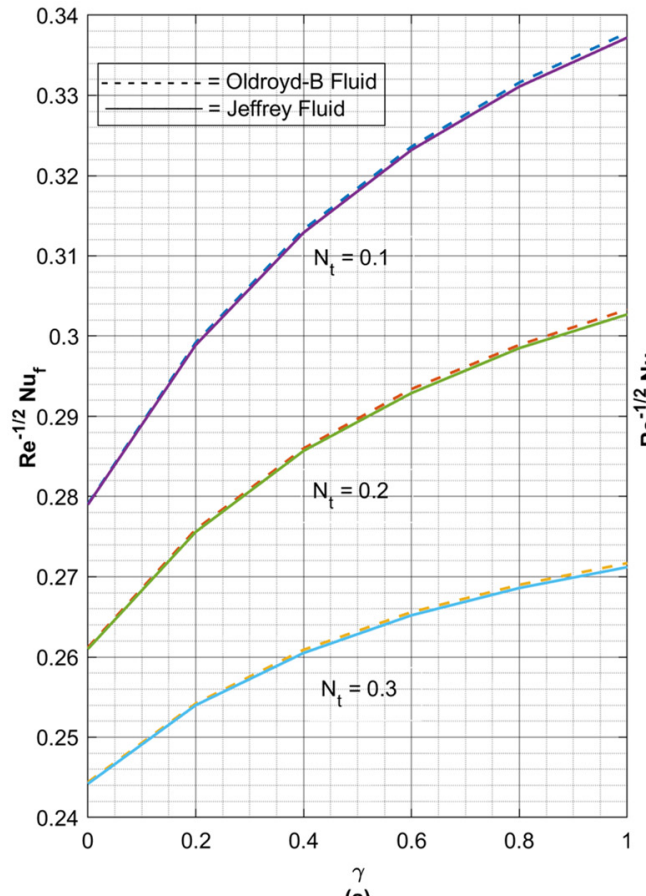

(a)

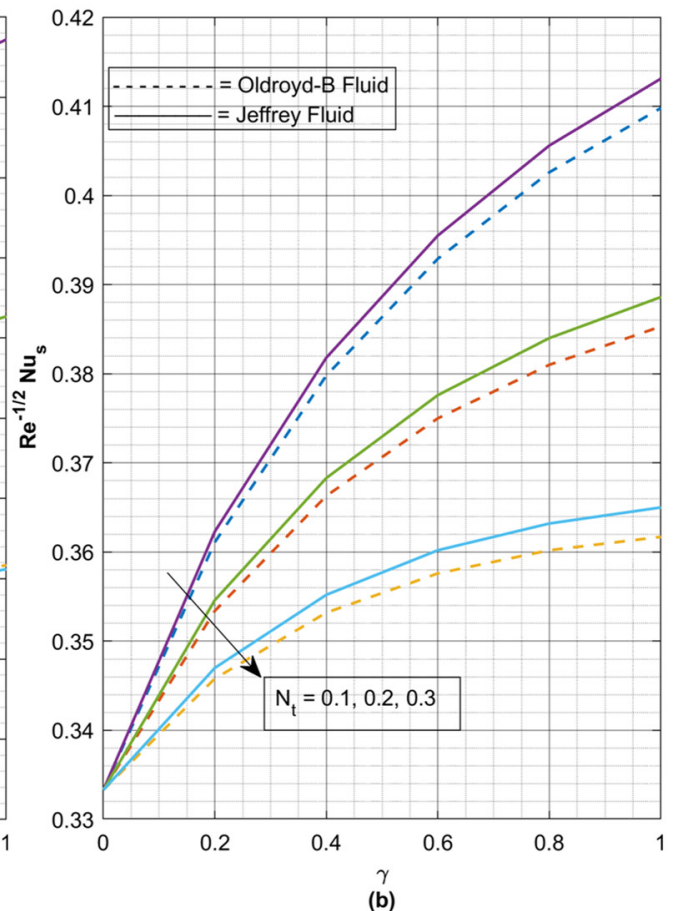

(b)

Figure 8. (a) Impact of $N_{t}$ and $\gamma$ on fluid phase Nusselt number and (b) Impact of $N_{t}$ and $\gamma$ on solid phase Nusselt number.

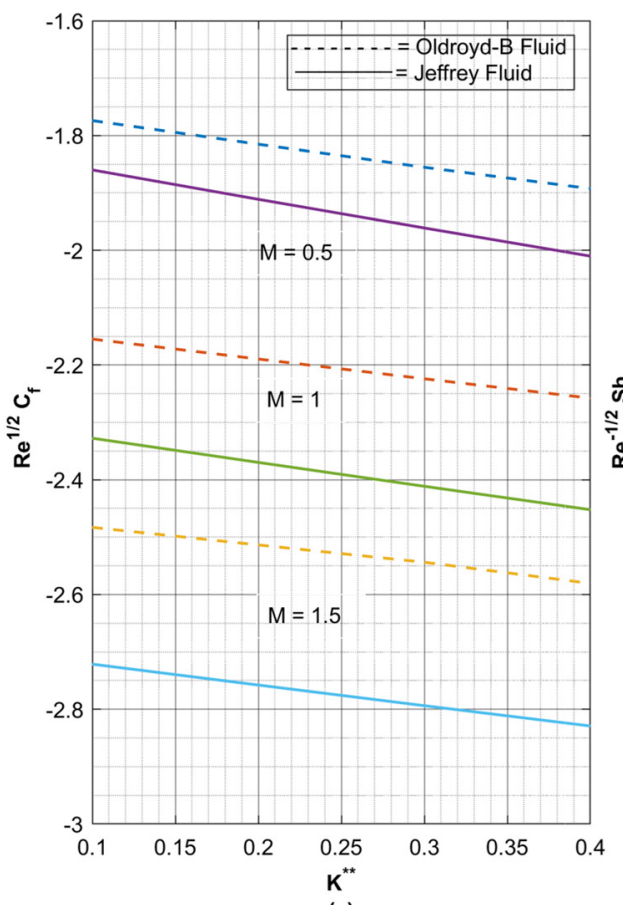

(a)

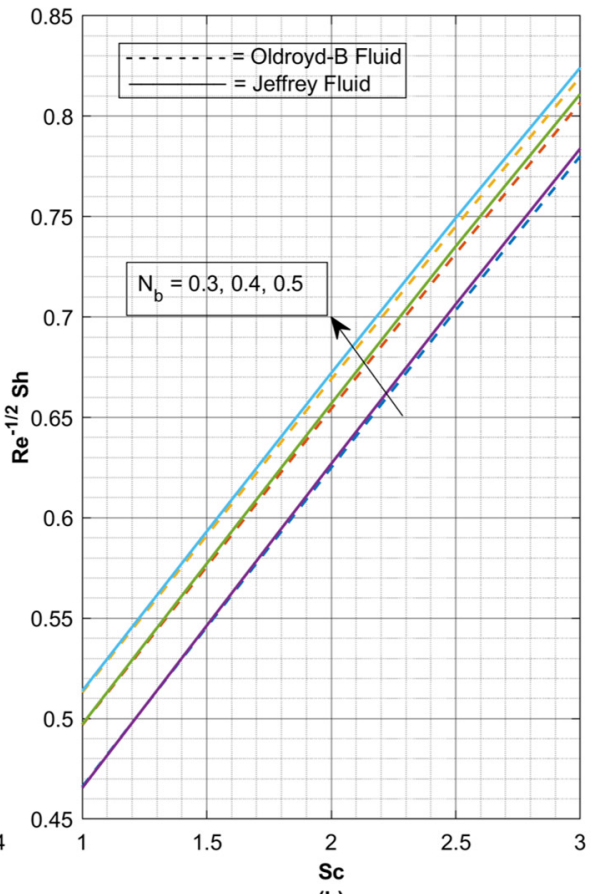

(b)

Figure 9. (a) Plot for skin friction and (b) Plot for Sherwood number.

\section{Conclusions}

Here, a mathematical model and theoretical study is performed to investigate the flow, heat and mass transfer behaviour of a non-Newtonian (Oldroyd-B and Jeffrey) liquid over 
an SS in a porous medium using LTNE approach. Further, a similar solution is given for steady two-dimensional flow subject to Buongiorno's theory to investigate the nature of MHD. The governing equations of the flow are reduced and cracked by employing R-K based shooting method. The key findings are as follows:

* The velocity of the Oldroyd-B fluid declines faster and high heat transfer is seen for lower values of magnetic parameter when compared to Jeffry fluid. However, for higher values of magnetic parameter, velocity of the Jeffery fluid declines faster and shows high heat transfer when compared to Oldroyd-B fluid.

* The liquid and solid phase heat transfer of Jefferey liquid is more than that of liquid and solid phase heat transfer of Oldroyd-B liquid and decays slowly for growing values of $\gamma$.

* The thermal gradient of the solid phase of both liquids drops and liquid phase of both liquids increases as $H$ increases.

* Jeffery liquid shows higher fluid phase heat transfer than Oldroyd-B liquid for rising values of Brownian motion and thermophoresis parameters.

* The rising values of thermophoresis parameter declines the liquid and solid phase heat transfer rate of both liquids.

Author Contributions: Conceptualization, B.C.P. and I.E.S.; methodology, B.C.P. and K.S.; software, R.J.P.G. and R.N.K.; validation, B.C.P. and I.E.S.; formal analysis, R.J.P.G. and R.N.K.; investigation, K.S.; resources, I.E.S.; data curation, B.C.P.; writing—original draft preparation, R.J.P.G. and R.N.K.; writing—review and editing, I.E.S. and B.C.P.; visualization, R.J.P.G. and R.N.K.; supervision, B.C.P. and I.E.S.; project administration, I.E.S.; funding acquisition, N/A. All authors have read and agreed to the published version of the manuscript.

Funding: This research received no external funding.

Institutional Review Board Statement: Not applicable.

Informed Consent Statement: Not applicable.

Conflicts of Interest: The authors declare no conflict of interest.

\section{Nomenclature}

$(u, v) \quad$ velocity components

$\varepsilon \quad$ porosity

$U_{w} \quad$ stretching velocity

$\mu \quad$ dynamic viscosity

$k$ thermal conductivity

$B_{0} \quad$ magnetic field

$\rho C_{p} \quad$ specific heat capacity

$D_{B} \quad$ Brownian diffusion co-efficient

$v_{f} \quad$ kinematic viscosity

$\theta_{f}(\eta) \quad$ fluid phase temperature profile

$K^{* *} \quad$ porosity parameter

$H$ non-dimensional inter-phase heat transfer parameter

$\lambda_{1} \quad$ Deborah number with respect to relaxation time

C concentration

$\lambda_{2}$ Deborah number with respect to retardation time

$D_{T} \quad$ thermophoresis diffusion co-efficient

$N u_{s} \quad$ Nusselt number for solid phase

$\tau \quad$ ratio of the effective heat capacity

$\gamma \quad$ porosity-modified conductivity ratio

Re local Reynolds number

M magnetic parameter

Sh Sherwood number

$C_{f} \quad$ skin friction 


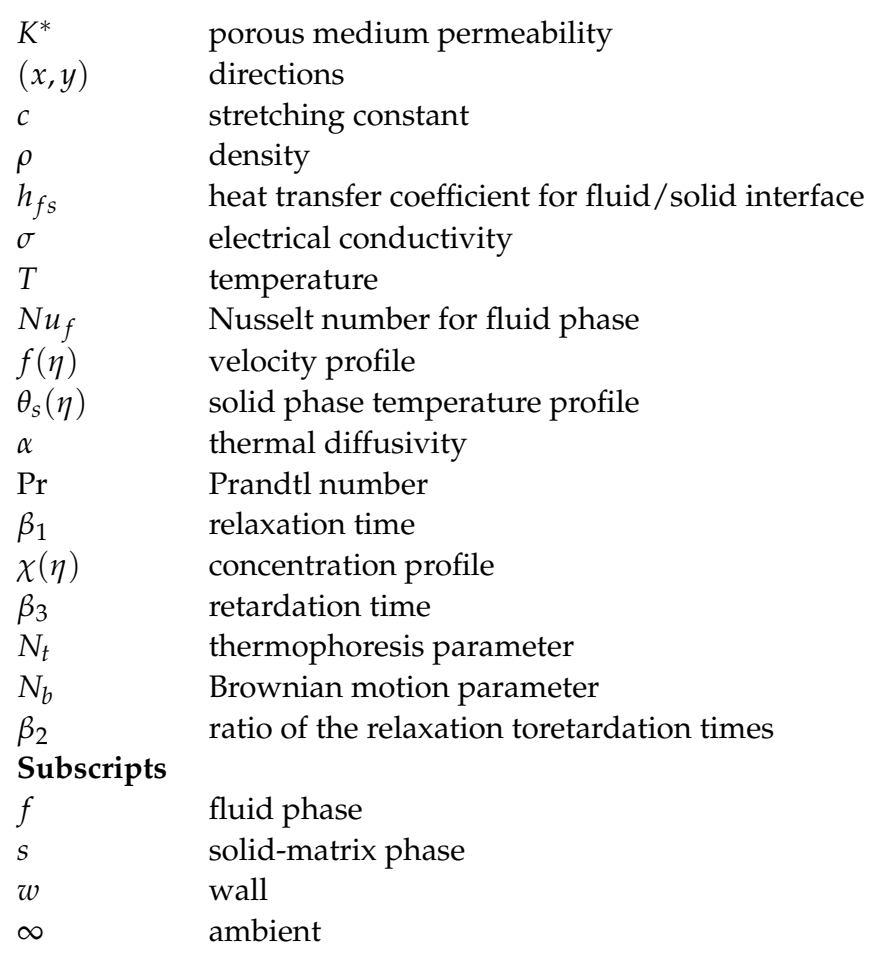

\section{References}

1. Amiri, A.; Vafai, K.; Kuzay, T.M. Effects of Boundary Conditions on Non-Darcian Heat Transfer Through Porous Media and Experimental Comparisons. Numer. Heat Transfer Part A Appl. 1995, 27, 651-664. [CrossRef]

2. Malashetty, M.; Shivakumara, I.; Kulkarni, S. The onset of Lapwood-Brinkman convection using a thermal non-equilibrium model. Int. J. Heat Mass Transf. 2005, 48, 1155-1163. [CrossRef]

3. Muthtamilselvan, M.; Prakash, D.; Doh, D.-H. Effect of thermal non-equilibrium on transient hydromagnetic flow over a moving surface in a nanofluid saturated porous media. J. Mech. Sci. Technol. 2014, 28, 3709-3718. [CrossRef]

4. Prakash, D.; Muthtamilselvan, M.; Niu, X.-D. Unsteady MHD non-Darcian flow over a vertical stretching plate embedded in a porous medium with thermal non-equilibrium model. Adv. Appl. Math. Mech. 2016, 8, 52-66. [CrossRef]

5. Hashemi, H.; Namazian, Z.; Zadeh, S.M.H.; Mehryan, S. MHD natural convection of a micropolar nanofluid flowing inside a radiative porous medium under LTNE condition with an elliptical heat source. J. Mol. Liq. 2018, 271, 914-925. [CrossRef]

6. Hayat, T.; Javed, T.; Abbas, Z. MHD flow of a micropolar fluid near a stagnation-point towards a non-linear stretching surface. Nonlinear Anal. Real World Appl. 2009, 10, 1514-1526. [CrossRef]

7. Mabood, F.; Yusuf, T.A.; Sarris, I.E. Entropy generation and irreversibility analysis on free convective unsteady MHD Casson fluid flow over a stretching sheet with Soret/Dufour in porous media. STRPM 2020, 11. [CrossRef]

8. Aslani, K.-E.; Mahabaleshwar, U.S.; Singh, J.; Sarris, I.E. Combined Effect of Radiation and Inclined MHD Flow of a Micropolar Fluid Over a Porous Stretching/Shrinking Sheet with Mass Transpiration. Int. J. Appl. Comput. Math. 2021, 7, 60. [CrossRef]

9. Kumar, R.S.V.; Dhananjaya, P.G.; Kumar, R.N.; Gowda, R.J.P.; Prasannakumara, B.C. Modeling and theoretical investigation on Casson nanofluid flow over a curved stretching surface with the influence of magnetic field and chemical reaction. Int. J. Comput. Methods Eng. Sci. Mech. 2021, 1-8. [CrossRef]

10. Radhika, M.; Gowda, R.J.P.; Naveenkumar, R.; Siddabasappa;Prasannakumara, B.C. Heat transfer in dusty fluid with suspended hybrid nanoparticles over a melting surface. Heat Transf. 2021, 50, 2150-2167. [CrossRef]

11. Buongiorno, J. Convective Transport in Nanofluids. J. Heat Transf. 2005, 128, 240-250. [CrossRef]

12. Chu, Y.-M.; Khan, M.I.; Khan, N.B.; Kadry, S.; Khan, S.U.; Tlili, I.; Nayak, M. Significance of activation energy, bio-convection and magnetohydrodynamic in flow of third grade fluid (non-Newtonian) towards stretched surface: A Buongiorno model analysis. Int. Commun. Heat Mass Transf. 2020, 118, 104893. [CrossRef]

13. Ijaz, M.; Ayub, M.; Malik, M.Y.; Khan, H.; A Alderremy, A.; Aly, S. Entropy analysis in nonlinearly convective flow of the Sisko model in the presence of Joule heating and activation energy: The Buongiorno model. Phys. Scr. 2020, 95, 025402. [CrossRef]

14. Gowda, R.P.; Kumar, R.N.; Prasannakumara, B.; Nagaraja, B.; Gireesha, B. Exploring magnetic dipole contribution on ferromagnetic nanofluid flow over a stretching sheet: An application of Stefan blowing. J. Mol. Liq. 2021, 335, 116215. [CrossRef]

15. Gowda, R.J.P.; Baskonus, H.M.; Kumar, R.N.; Prasannakumara, B.C.; Prakasha, D.G. Computational Investigation of Stefan Blowing Effect on Flow of Second-Grade Fluid Over a Curved Stretching Sheet. Int. J. Appl. Comput. Math. 2021, 7, 109. [CrossRef]

16. Lund, L.A.; Omar, Z.; Khan, I. Darcy-Forchheimer porous medium effect on rotating hybrid nanofluid on a linear shrinking/stretching sheet. Int. J. Numer. Methods Heat Fluid Flow 2021. ahead-of-print. [CrossRef] 
17. Irfan, M.; Farooq, M.A.; Mushtaq, A.; Shamsi, Z.H. Unsteady MHD Bionanofluid Flow in a Porous Medium with Thermal Radiation near a Stretching/Shrinking Sheet. Math. Probl. Eng. 2020, 2020, e8822999. [CrossRef]

18. Gowda, R.P.; Kumar, R.N.; Jyothi, A.; Prasannakumara, B.; Sarris, I. Impact of Binary Chemical Reaction and Activation Energy on Heat and Mass Transfer of Marangoni Driven Boundary Layer Flow of a Non-Newtonian Nanofluid. Processes 2021,9 , 702. [CrossRef]

19. Kumar, R.N.; Gowda, R.J.P.; Gireesha, B.J.; Prasannakumara, B.C. Non-Newtonian hybrid nanofluid flow over vertically upward/downward moving rotating disk in a Darcy-Forchheimer porous medium. Eur. Phys. J. Spé. Top. 2021. [CrossRef]

20. Tadesse, F.B.; Makinde, O.D.; Enyadene, L.G. Mixed Convection of a Radiating Magnetic Nanofluid past a Heated Permeable Stretching/Shrinking Sheet in a Porous Medium. Math. Probl. Eng. 2021, 2021, e6696748. [CrossRef]

21. Sandeep, N.; Kumar, B.R.; Kumar, M.J. A comparative study of convective heat and mass transfer in non-Newtonian nanofluid flow past a permeable stretching sheet. J. Mol. Liq. 2015, 212, 585-591. [CrossRef]

22. Sandeep, N.; Sulochana, C. Momentum and heat transfer behaviour of Jeffrey, Maxwell and Oldroyd-B nanofluids past a stretching surface with non-uniform heat source/sink. Ain Shams Eng. J. 2018, 9, 517-524. [CrossRef]

23. Reddy, G.K.; Yarrakula, K.; Raju, C.S.K.; Rahbari, A. Mixed convection analysis of variable heat source/sink on MHD Maxwell, Jeffrey, and Oldroyd-B nanofluids over a cone with convective conditions using Buongiorno's model. J. Therm. Anal. Calorim. 2018, 132, 1995-2002. [CrossRef]

24. Almakki, M.; Nandy, S.K.; Mondal, S.; Sibanda, P.; Sibanda, D. A model for entropy generation in stagnation-point flow of non-Newtonian Jeffrey, Maxwell, and Oldroyd-B nanofluids. Heat Transf.-Asian Res. 2019, 48, 24-41. [CrossRef]

25. Saeed, A.; Islam, S.; Dawar, A.; Shah, Z.; Kumam, P.; Khan, W. Influence of Cattaneo-Christov Heat Flux on MHD Jeffrey, Maxwell, and Oldroyd-B Nanofluids with Homogeneous-Heterogeneous Reaction. Symmetry 2019, 11, 439. [CrossRef]

26. Nield, D.A.; Bejan, A. Convection in Porous Media; Springer: New York, NY, USA, 2013.

27. Ishak, A.; Nazar, R.; Pop, I. Boundary layer flow and heat transfer over an unsteady stretching vertical surface. Meccanica 2009, 44, 369-375. [CrossRef]

28. Vajravelu, K.; Prasad, K.V.; Ng, C.-O. Unsteady convective boundary layer flow of a viscous fluid at a vertical surface with variable fluid properties. Nonlinear Anal. Real World Appl. 2013, 14, 455-464. [CrossRef] 